Acta vet. seand. $1971,12,300-302$.

Brief Communication

\title{
RUMINAL METABOLISM AND METHEMOGLOBIN-FORMING EFFECT OF DINOBUTON IN SHEEP
}

Many aromatic nitro compounds are reduced to the corresponding amines in the rumen by the activity of the rumen flora, and this may influence the effect of such compounds when given orally to ruminants. The dinitrophenol herbicide DNBP (2-(1methyl-n-propyl)-4,6-dinitrophenol, dinoseb) is a good example of a compound with this property, as DNBP has a methemoglobin-forming effect in ruminants (Frøslie \& Karlog 1970). These authors connected this phenomenon with the ruminal metabolism of DNBP, because it is reduced to 6-ANBP (2-(1-methyl-npropyl)-4-nitro-6-aminophenol), and 6-ANBP successively is reduced to DABP (2-(1-methyl-n-propyl)-4,6-diaminophenol) in the rumen.

Dinobuton (2-(1-methyl-n-propyl)-4,6-dinitrophenyl isopropyl carbonate), an ester of DNBP, is also used in agriculture. This paper will present investigations into the metabolism of dinobuton in fresh rumen fluid in vitro and an experiment to demonstrate a possible methemoglobin-forming effect of dinobuton when given intraruminally to sheep.

\section{EXPERIMENTAL AND ANALYTICAL METHODS}

The experiments were carried out as described by Frøslie \& Karlog (1970) and Frøslie (in press). The analytical methods used by Frøslie \& Karlog were modified and combined with the methods given by Matsuo \& Casida (1970). The methanolic supernatants after centrifugation of the samples (Frøslie \& Karlog) were evaporated in vacuum, the residue dissolved in benzene: methanol 1:1, and transferred to the aluminium oxide columns. Dinobuton, DNBP, and 6-ANBP were then eluted from the columns with benzene, methanol, and $0.1 \mathrm{~N}-\mathrm{NaOH}$, respectively. The two last mentioned compounds were determined as described by Fr $\phi$ slie \& Karlog, while dinobuton was determined as DNBP after evaporation of the benzene eluates and hydrolysis 
in $0.1 \mathrm{~N}$ methanolic $\mathrm{NaOH}$. TLC were also used for identification of the metabolites.

\section{RESUL'TS}

The results of one of the experiments in vitro are given in Fig. 1. Dinobuton, at concentrations of $150 \mu \mathrm{g} / \mathrm{ml}\left(4.6 \times 10^{-4} \mathrm{M}\right)$, is rapidly converted in fresh rumen fluid at $\mathrm{pH}$ 6.8. Most of the compound added is decomposed during the first half hour, and simultaneously increasing amounts of 6-ANBP were found. Ten min. after administration this metabolite amounted to $10 \%$ of the dinobuton added, and after $1 / 2 \mathrm{hr}$. it amounted to $66 \%$. Then the concentration of 6-ANBP decreased and after $3 \mathrm{hrs}$. only $10 \%$ were left. In the first 2 samples of rumen fluid small amounts of DNBP were found. Heating of the rumen fluid to $100^{\circ} \mathrm{C}$ for $1 / 2 \mathrm{hr}$. (in a closed bottle) destroyed its degradation capacity almost completely. Intra ruminal application of $2.65 \mathrm{~g}$

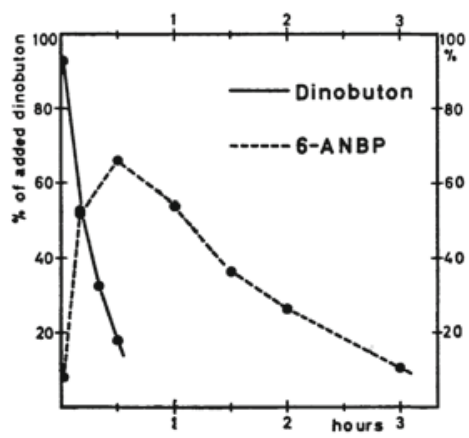

F i g u r e 1. Metabolism of dinobuton in rumen fluid $(\mathrm{pH} 6.8)$ in vitro. $100 \%=150 \mu \mathrm{g} / \mathrm{ml}$.

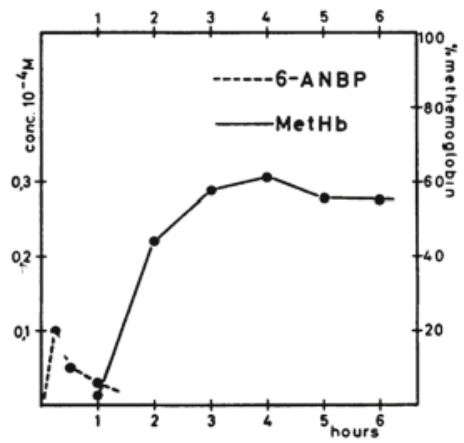

Figure 2. Methemoglobin concentrations and concentrations of 6-ANBP in plasma in a sheep after intraruminal application of dinobuton. Dose: $68 \mathrm{mg} / \mathrm{kg}$.

of dinobuton to a sheep of $39 \mathrm{~kg}(68 \mathrm{mg} / \mathrm{kg}$, corresponding to $50 \mathrm{mg}$ DNBP $/ \mathrm{kg}$ ) lead to a serious methemoglobinemia, as seen in Fig. 2. After 4 hrs. the methemoglobin concentration was $61 \%$ (of total $10.5 \mathrm{~g} \mathrm{Hb} / 100 \mathrm{ml}$ blood). 'The methemoglobinemia lasted for several days, and hemolysis occurred and caused a marked drop in the hemoglobin concentration. The sheep died after 4 days. In the first hour after administration small amounts of 6-ANBP were found in plasma ( $\max$. value $0.1 \times 10^{-4} \mathrm{M}$ ), but dinobuton, DNBP, or 6-AcANBP (the N-acetylated 6-ANBP) could not be detected in plasma samples. 


\section{DISCUSSION}

Degradation of dinitrophenyl esters by hydrolysis may occur in the organism of animals (Ernst \& Bür 1964), on plants as a photodecomposition (Matsuo \& Casida 1970), or as a result of pure chemical reactions (alkaline hydrolysis, Fieser \& Fieser 1956). The experiments presented here show that dinobuton is hydrolyzed to DNBP in fresh rumen fluid too, as a result of the activity of the rumen flora. DNBP, in turn, is reduced to its amino metabolites, in agreement with previous findings (Fr $\phi$ slie \& Karlog 1970). It is therefore logical that dinobuton like DNBP exhibit a methemoglobin-forming effect when given intraruminally, and the results presented here confirm that this is the case.

DNBP and 6-ANBP, at doses of $50 \mathrm{mg} / \mathrm{kg}$, both lead to a methemoglobinemia of about $50 \%$ when given intraruminally to sheep (Frøslie 1971, Frøslie in press). It may then be concluded that dinobuton, DNBP, and 6-ANBP all show the same effect when given intraruminally to sheep, because they are metabolized in the same way. The end product of this ruminal metabolism, the diaminophenol DABP, is pointed out as the possible inducer of methemoglobinemia by DNBP poisoning in ruminants (Fr $\phi$ slie in press).

Arne Frøslie

The National Veterinary Institute,

Oslo, Norway.

\section{REFERENCES}

Ernst, W.\&F. Bär: Die Umvandlung des 2,4-Dinitro-6-sec.-butylphenols und seiner Ester im tierischen Organismus. (The metabolism of 2,4-dinitro-6-sec.-butylphenol and its esters in the organism of animals.) Arzneimittel-Forsch. 1964, 14, 81-84.

Fieser, L. F. \& M. Fieser: Organic Chemistry. 3rd Ed., Reinhold, New York 1956, p. 178.

Frøslie, A.: The difference in methemoglobin formation by 6 -amino metabolites of DNOC and DNBP in ruminant and nonruminant animals, with special references to sheep and swine. (In press).

Frøslie, A.: 1971. Not published.

Frøslie, A. \& O. Karlog: Ruminal metabolism of DNOC and DNBP. Acta vet. scand. $1970,11,114-132$.

Matsuo, H. \& J. E. Casida: Photodegradation of two dinitrophenolic pesticide chemicals, dinobuton and dinoseb, applied to bean leaves. Bull. envir. Contam. Toxicol. 1970, 5, 72-78. 